\title{
Hemangioma lobular capilar lingual. Reporte de un caso
}

\section{Caso Clínico}

Mónica Astudillo Ramírez 1,a, Rubén Soto Galaz 2,a, Carla Pia Cittadini ${ }^{3, a}$, Rodrigo Bravo Ahumada ${ }^{2, b}$, Marcelo Mardones Muñoz ${ }^{4,6}$

${ }^{1}$ Universidad Estatal de Guayaquil, Hospital Clínico San José, Equipo Cirugía Maxilofacial, Santiago, Chile.

${ }^{2}$ Universidad de Chile, Hospital Clínico San José, Equipo Cirugía Maxilofacial, Santiago, Chile.

${ }^{3}$ Universidad Mayor, Hospital Clínico San José, Equipo Cirugía Maxilofacial, Santiago, Chile.

${ }^{4}$ Universidad de Chile, Hospital Clínico San José, Servicio de Cirugía Maxilofacial, Santiago, Chile.

${ }^{\text {a }}$ Cirujano Dentista.

\section{Lingual lobular capillary hemangioma. A case report}

\section{Resumen}

El hemangioma lobular capilar es una hiperplasia inflamatoria en respuesta a una irritación crónica, la cual da lugar a una lesión exofítica eritematosa debido a la proliferación de tejido fibrovascular. En la boca puede verse en cualquier ubicación, pero es más frecuente en la zona gingival. Se reporta el caso clínico de una paciente embarazada de 38 años que consultó al equipo de Cirugía Maxilofacial del Hospital San José al presentar una lesión tumoral compatible con hemangioma lobular capilar en cara lingual lateral derecha, diagnóstico confirmado con histopatología. El objetivo de este reporte es especificar características y tratamiento de dicha lesión con localización infrecuente.

Palabras clave: Granuloma piogénico; Hemangioma capilar lobular; Lengua; Embarazo (fuente: DeCS BIREME).

\begin{abstract}
Lobular capillary hemangioma is an inflammatory hyperplasia in response to chronic irritation, resulting in an exophytic erythematous lesion due to proliferation of fibrovascular tissue. Intraorally, it can appear in any location, but it is more frequent in the gingival region. This article describes a case of a 38-year-old pregnant woman who attended the Maxillofacial Surgery Unit at San José Hospital, presenting a tumoral lesion on the right lateral area of the tongue compatible with a lobular capillary hemangioma. The diagnosis was confirmed with histopathology. The aim of this report is to specify the characteristics and treatment of a lesion with infrequent location.
\end{abstract}

Keywords: Pyogenic granuloma; Lobular capillary hemangioma; Tongue; Pregnancy (source: MeSH NLM).

\section{Correspondencia:}

Mónica Astudillo Ramírez: odmonica.astudillo@gmail.com Av. San José 1196, Independencia, Santiago de Chile. Código postal: 9480000

ORCID: 0000-0001-8069-4038

\section{Coautores:}

Rubén Soto Galaz: ruben.soto@odontologia.uchile.cl ORCID: 0000-0002-2055-2641

Carla Pia Cittadini: odcarlacittadini@gmail.com ORCID: 0000-0002-1541-0665

Marcelo Mardones Muñoz: drmardones@gmail.com ORCID: 0000-0003-4936-3294

Rodrigo Bravo Ahumada: rbravo14@gmail.com ORCID: 0000-0002-3480-1215

Editora:

María Eugenia Guerrero

Universidad Nacional Mayor de San Marcos, Perú.

Conflicto de intereses: los autores declaran no tener conflictos de interés.

Fuente de financiamiento: autofinanciado.

Recibido: 09/02/21

Aceptado: 22/04/21

Publicado: 01/07/21

(c) Los autores. Este artículo es publicado por la revista Odontología Sanmarquina de la Facultad de Odontología, Universidad Nacional Mayor de San Marcos. Este es un artículo de acceso abierto, distribuido bajo los términos de la licencia Creative Commons Atribución 4.0 Internacional (CC BY 4.0) [https://creativecommons.org/ licenses/by/4.0/deed.es] que permite el uso, distribución y reproducción en cualquier medio, siempre que la obra original sea debidamente citada de su fuente original. 


\section{Introducción}

El hemangioma lobular capilar (HLC) es una lesión de tipo benigna reactiva, exuberante y excesiva del tejido conectivo. El término granuloma piogénico es erróneo, ya que no hay producción de pus como lo haría suponer el adjetivo piogénico. Sin embargo, es un tumor por granulación como lo indica el nombre granuloma. Otros nombres utilizados para esta tumoración benigna son: granuloma del embarazo, épulis angiomatoso, enfermedad de Crocker-Hartzell, e hiperplasia nodular gingival ${ }^{1}$.

La causa precisa para el desarrollo es desconocida, sin embargo, se cree que es una respuesta exagerada de los tejidos a traumas de baja intensidad. Los principales factores desencadenantes son irritación gingival y la inflamación debido a la falta de higiene oral. También se asocia a cambios hormonales durante la pubertad y el embarazo, así como en mujeres que tienen antecedentes de administración de anticonceptivos orales ${ }^{2}$.

En el embarazo podemos encontrar un incremento de la producción de ciertas hormonas como son los estrógenos, la progesterona, gonadotropinas entre otras. Este aumento de hormonas tiene una relación directa con la aparición de ciertas patologías reversibles y transitorias en la cavidad bucal, dentro de las cuales la más frecuente es el hemangioma lobular capilar ${ }^{3}$. El HLC se presenta en 1\% de las embarazadas, principalmente durante el segundo y tercer trimestre, y aparece mayoritariamente en la encía ${ }^{4}$.

Puede encontrarse intra o extraoralmente. Extraoralmente puede afectar la piel del cuello, cara, extremidades superiores e inferiores, membranas mucosas de nariz y párpados 5 .

Dentro de sus características clínicas presenta coloración roja, a menudo presentan una seudomembrana grisácea sobre su superficie secundaria a la ulceración del epitelio ${ }^{6}$. Algunas veces se ulceran a causa de un traumatismo secundario y un componente infeccioso inespecífico, pueden presentar superficie lisa o lobular, consistencia blanda y friable propensa al sangrado ante el traumatismo masticatorio o el cepillado ${ }^{7}$.

Generalmente se observa en mucosa queratinizada, ya sea en encía marginal o papila interdental, también se puede extender a otras regiones de la cavidad bucal, pudiendo extenderse desde la encía bucal a la lingual o la palatina; no obstante, generalmente se limitan a la superficie vestibular ${ }^{8}$. Debido a la localización inusual de esta lesión en lengua, nos parece importante mencionar este caso clínico, con el objetivo de recordar a los profesionales de la salud que se puede observar este tipo de hemangioma en regiones anatómicas poco usuales.

\section{Reporte del caso}

Diagnóstico y etiología. Paciente embarazada de 38 años, acude a la Unidad de Cirugía Maxilofacial del Hospital Clínico San José en Santiago de Chile por presentar lesión en borde lateral derecho de la lengua, indolora, de aproximadamente tres meses de evolución. Paciente consulta por primera vez durante el tercer trimestre de embarazo, como antecedente mórbido presenta diabetes gestacional. Relata que presentó un episodio de sangrado lingual, hecho que dio a conocer la presencia de la lesión.

Al examen clínico intraoral se observaron líneas albas (morsicatio buccarum) en mucosa yugal, e higiene oral deficiente. Junto con esto se pesquisó un aumento de volumen en zona lingual lateral derecha de 14x9x8 mm, de consistencia firme, coloración rojiza, y base sésil ( $\mathrm{Fi}$ gura 1). El diagnóstico presuntivo de esta lesión fue hemangioma lobular capilar lingual. Se programa cirugía posterior al parto.

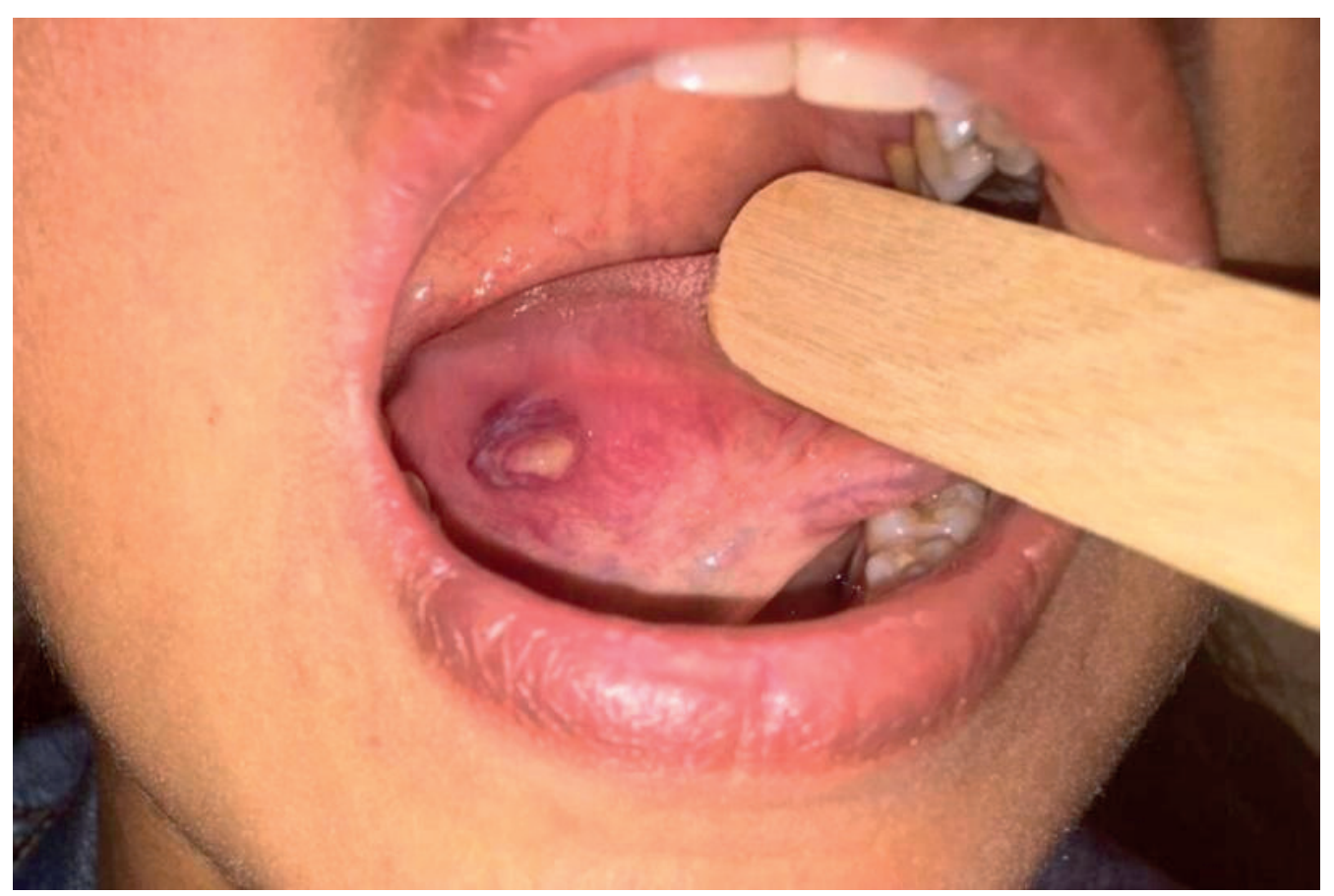

Figura 1. Inspección de la lesión 
Tratamiento. Bajo anestesia local con vasoconstrictor más sedación endovenosa, se realizó biopsia escisional de la lesión con bisturí frío, sin necesidad de cauterización en la base de la lesión; se realiza hemostasia local, y se sutura lecho quirúrgico con material reabsorbible (Vicryl ${ }^{\mathrm{TM}}$ 4.0) en la mucosa lingual, sin complicaciones (Figuras 2,3 y 4). Cabe destacar que el uso de sedación endovenosa es manejo protocolario del recinto hospitalario con el objetivo de evitar movimientos involuntarios de parte del paciente y para mayor control operatorio frente a posibles efectos secundarios como sangrado excesivo, además de, incluir el consentimiento informado previo a la cirugía. Se indica como manejo post operatorio régimen blando más agua a tolerancia, reposo relativo, higiene oral prolija y analgesia con Paracetamol $500 \mathrm{mg}$ cada 8 horas, por 3 días.

Resultados del tratamiento. Como resultado del examen histopatológico se observa epitelio plano pluriestratificado paraqueratinizado con zonas hiperplásicas y ulceradas. Estas últimas contienen una capa de fibrina $y$, subyacente a esta, un infiltrado inflamatorio predominantemente agudo, destacando la presencia de neutrófilos. En los sectores de epitelio hiperplásico, el tejido

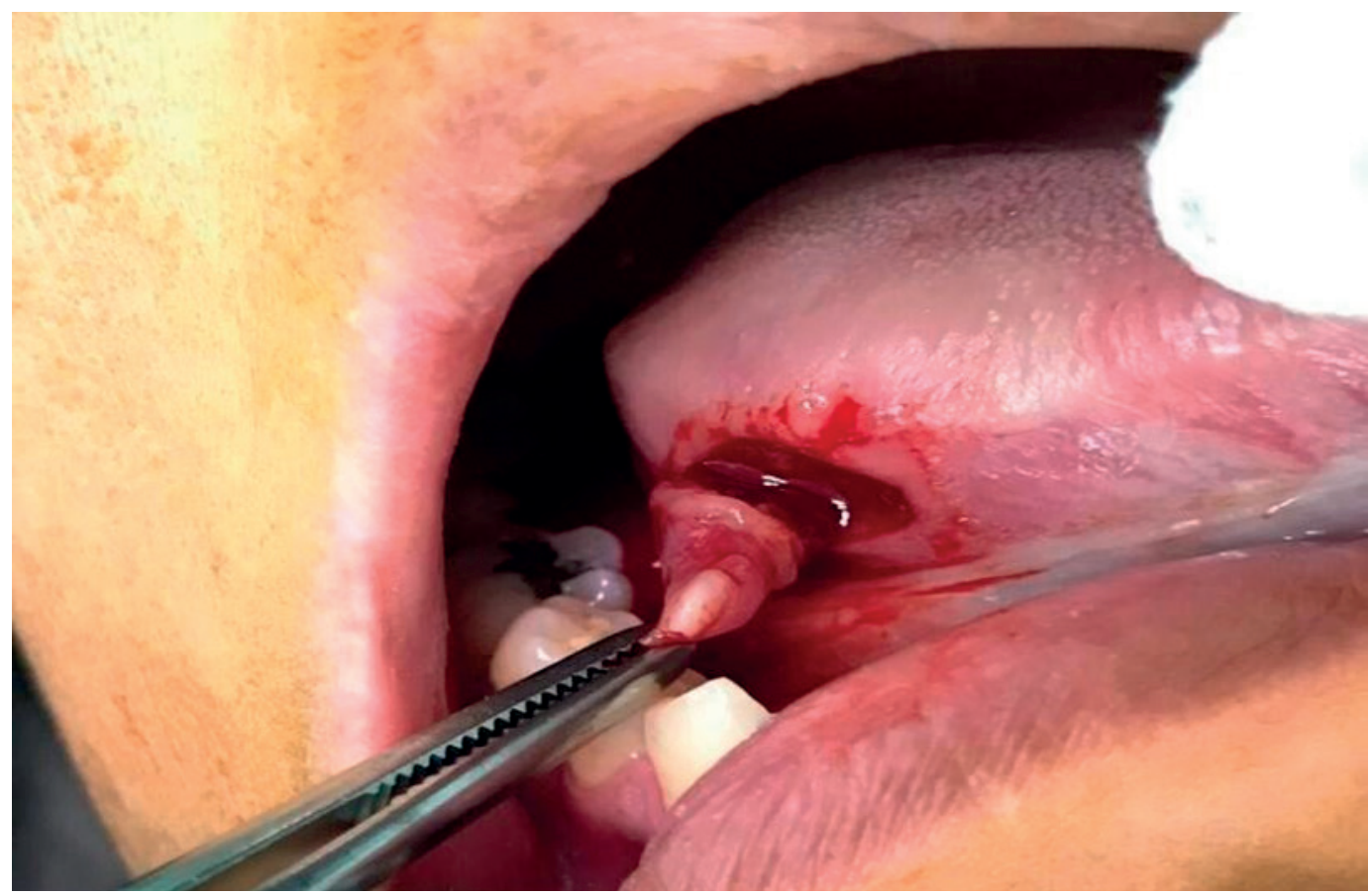

Figura 2. Exéresis quirúrgica de la lesión, mediante diéresis aguda

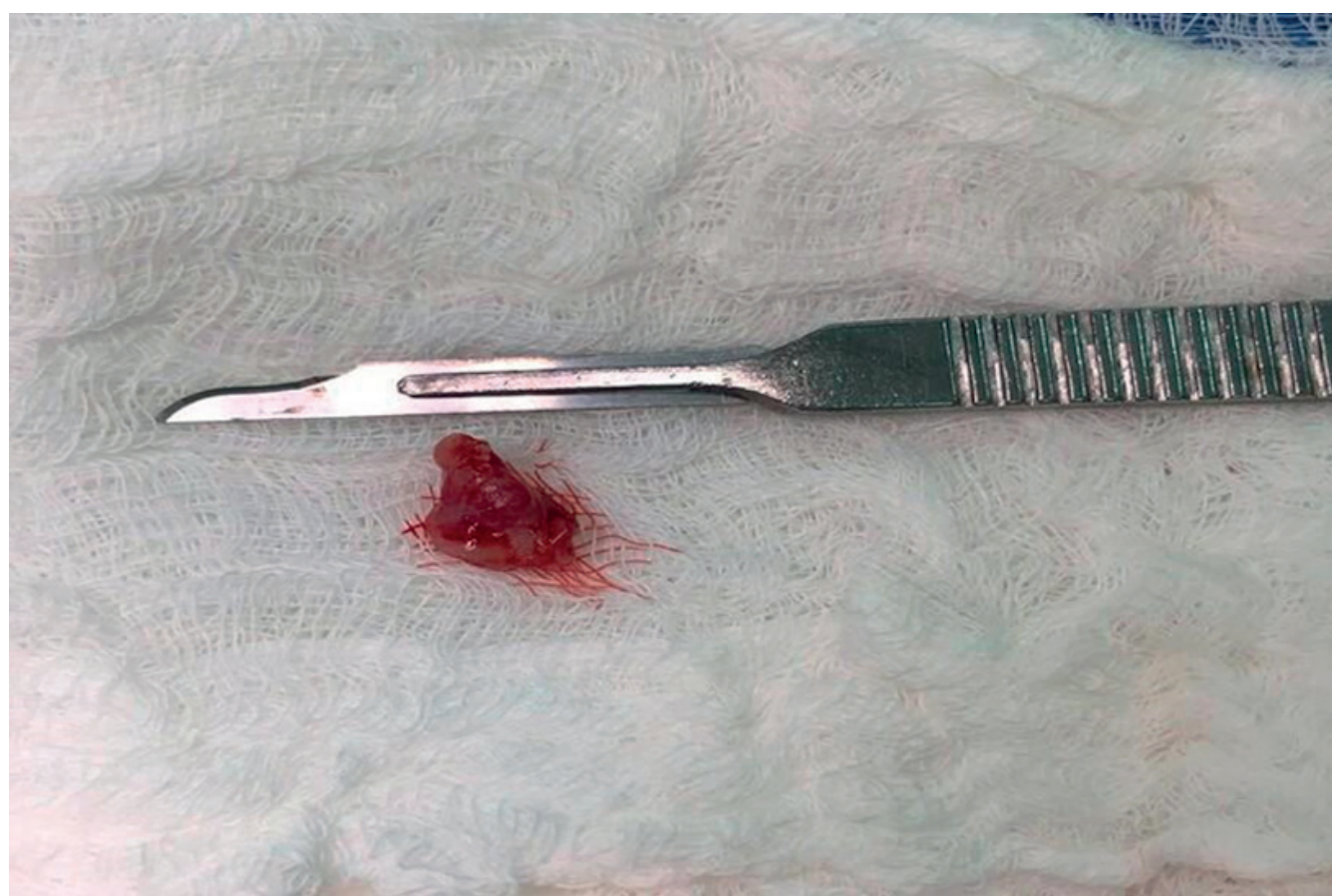

Figura 3. Pieza operatoria obtenida para estudio histopatológico 


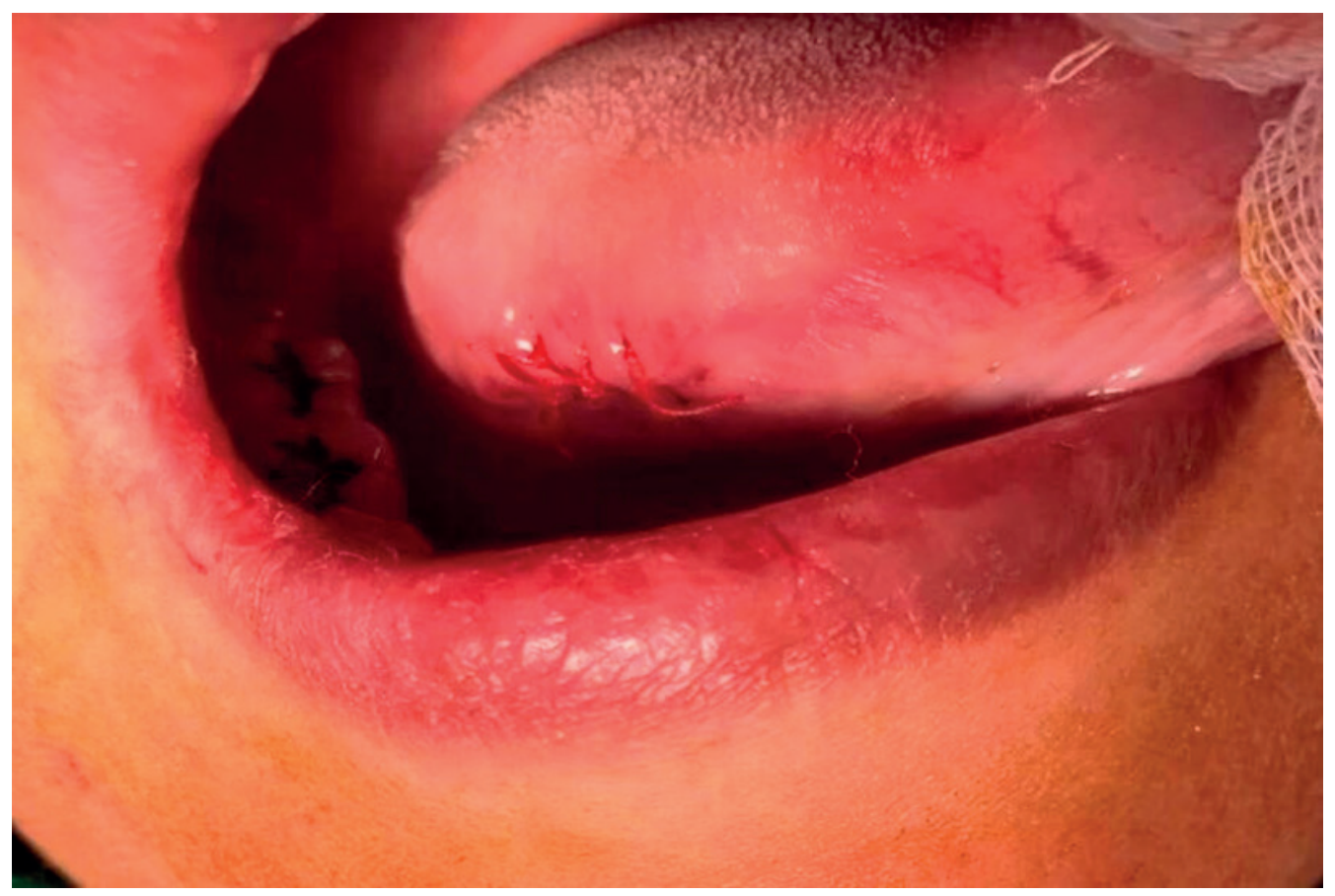

Figura 4. Síntesis del lecho quirúrgico

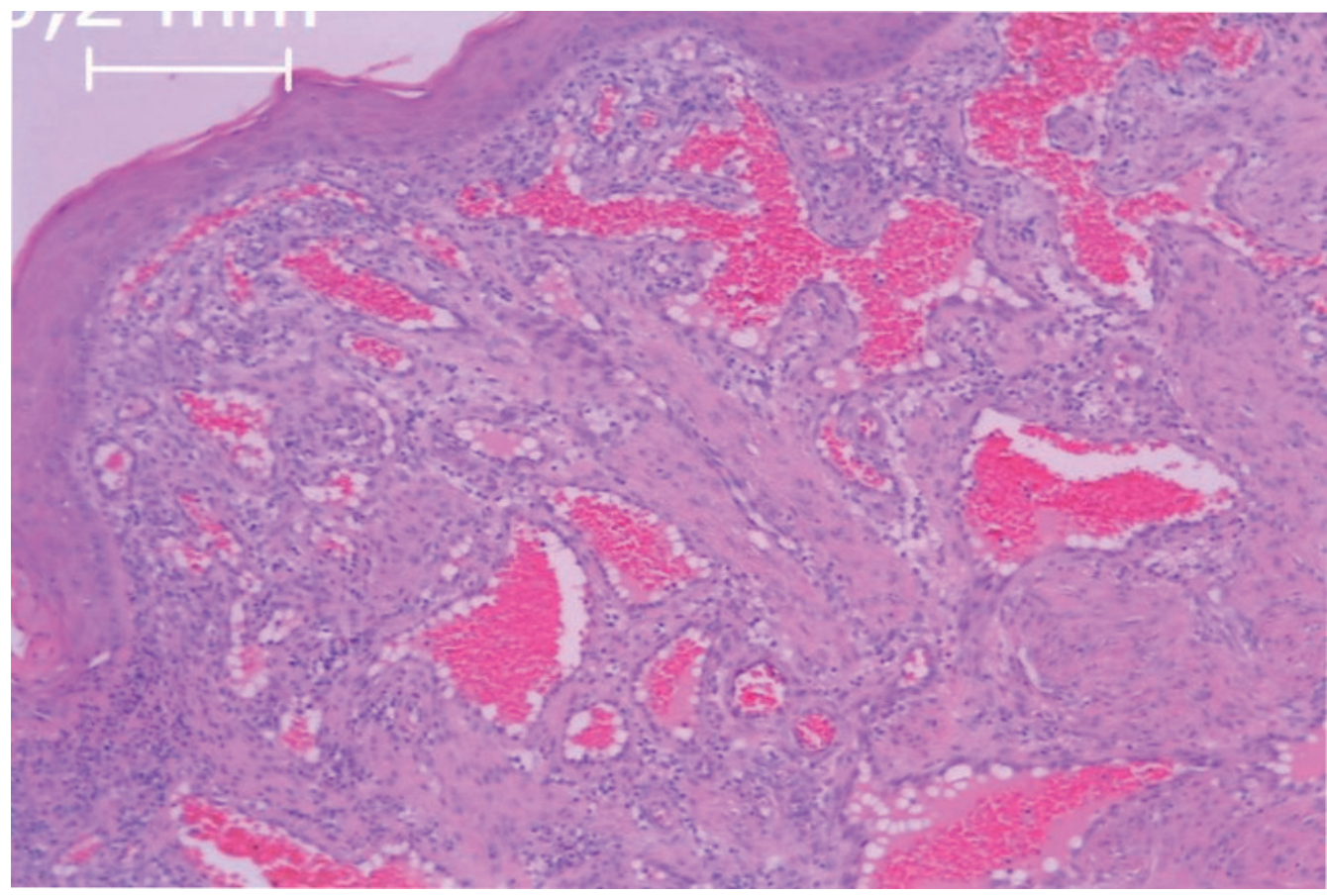

Figura 5. Corte histológico de la lesión. Zona superior: presencia de epitelio plano pluriestratificado paraqueratinizado con zonas hiperplásicas y ulceradas. Se observa presencia de vasos sanguíneos con abundantes glóbulos rojos, organizados en agregados lobulares propios de hemangioma lobular capilar

conjuntivo presenta un predominio fibroso, con menor cantidad de vasos sanguíneos que en la zona ulcerada. El diagnóstico anatomopatológico confirma lesión compatible con hemangioma lobular capilar (Figura 5).

\section{Discusión}

El término granuloma piógeno, como mayormente es conocido el hemangioma lobular, capilar fue descrito por primera vez en 1897 por Poncet y Dor, quienes lo denominaron botryomycosis hominis, a partir de lo cual se le asignan diferentes nombres tales como: hemangioma lobular capilar, granuloma del embarazo, tumor benigno vascular, épulis vascular, épulis angiomatoso y enfermedad de Crocker-Hartzell; estos últimos fueron quienes entre 1903 y 1904 introducen el término granuloma piógeno. En la actualidad se reconoce que este término está mal utilizado debido a que dicha lesión no se caracteriza por presencia de exudado purulento, ni 
se trata de un proceso infeccioso; por el contrario, encontramos tejido de proliferación fibrosa y vascular sin relación con bacterias productoras de pus ${ }^{8}$.

El HLC en el 75\% de los casos puede ubicarse en encía, sin embargo, también puede localizarse con menor prevalencia en labio inferior, lengua, paladar y mucosa bucal. Debido a que puede ubicarse en diferentes zonas de la cavidad oral, presenta varios diagnósticos diferenciales, dentro los cuales podemos encontrar las siguientes patologías con características clínicas similares: fibroma traumático, hemangioma, sarcoma de Kaposi, angiosarcoma, cáncer metastásico, y linfoma no Hodgkin, dando así importancia a realizar un diagnóstico correcto y oportuno. El estudio histopatológico determina el diagnóstico definitivo ${ }^{9}$.

La inflamación gingival exacerbada en mujeres embarazadas está bien documentada desde el punto de vista clínico e histológico, su etiología aún no se ha establecido con claridad y no se sabe por qué sólo algunas mujeres embarazadas desarrollan signos francos de inflamación gingival. Hoy en día se han propuesto cuatro posibles mecanismos, entre los que se incluyen un aumento de la permeabilidad vascular, un cambio a un fenotipo gingival más susceptible, una depresión del sistema inmunitario, y cambios en el biofilm sub o supragingival ${ }^{10}$.

Yuan et al. ${ }^{11}$ describen la relación entre el HLC y los factores angiogénicos en el embarazo; concluyen que las hormonas esteroideas femeninas mejoran la expresión de los factores angiogénicos en el tejido inflamado y disminuyen la apoptosis de las células en el HLC. Otros autores apoyan que el metabolismo de la progesterona desempeña un papel especial en la fisiología gingival en las mujeres embarazadas permitiendo un aumento en la reacción de los tejidos tipo crónico, lo que resulta clínicamente en una aparición exagerada de la inflamación ${ }^{11}$.

El tratamiento de esta lesión inflamatoria dependerá de su ubicación. Este consiste en la eliminación de cualquier factor irritante causante, seguido de la escisión mediante diversas técnicas convencionales con bisturí o electrocauterización, y no convencionales como crioterapia, escleroterapia, y terapia láser; esta última puede combinarse con inyección de etanol, la cual se considera en zonas altamente vascularizadas como la lengua para evitar riesgo de sangrado profuso ${ }^{12,13}$.

Entre aquellas técnicas que proporcionan un alto porcentaje de éxito y baja recidiva se incluye: criocirugía, láser, y la técnica convencional escisión quirúrgica con bisturí la cual puede ser realizada en una sola sesión siendo esta considerada una ventaja frente a las otras técnicas que requieren de varias sesiones, equipos y entrenamiento específico del personal ${ }^{14}$.

La terapia quirúrgica en la región anterior puede producir defectos mucogingivales no deseados, por esto al ser la zona de la cavidad oral que muestra mayor recidiva se debe elegir una técnica adecuada para una exéresis completa ${ }^{12}$. La recidiva puede observarse hasta en un
$16 \%$ de los casos, en estas lesiones reincidentes puede observarse múltiples nódulos profundos en el lugar original del tumor. Entre las causas probables de recidiva encontramos presencia de escisión incompleta, por no haber eliminado los factores etiológicos, o por una nueva lesión en la zona. Para evitar recurrencia por excisión incompleta se recomienda realizar márgenes de $2 \mathrm{~mm}$ en su periferia clínica llegando en profundidad hasta el periostio o restos en la base de la lesión original ${ }^{2,12}$.

El tratamiento de un HLC que recurre varias veces después de resecciones quirúrgicas y con la formación de lesiones satélites en encía superior fue descrito en el 2006 por Parisi y colaboradores. Este se basa en el uso de una serie de inyecciones de corticoides intralesionales. Se cree que las lesiones respondieron a las acciones antiinflamatorias y vasoconstrictoras de los corticosteroides, y pueden haber impedido o suprimido la liberación de factores angiogénicos 5 .

La histopatología de esta lesión presenta características comunes con otros casos similares reportados en la literatura, encontrándose así zonas ulceradas e hiperplásicas, presencia de fibrina, vasos sanguíneos de variados tamaños dispersos de manera lobulillar y neutrófilos. Otros elementos celulares y acelulares reportados en este tipo de lesión son capilares revestidos por células endoteliales, fibroblastos intercalados, fibras de colágeno y una densa infiltración celular inflamatoria crónica que puede contener células plasmáticas y linfocitos ${ }^{15-18}$.

En nuestro reporte de caso se logró la exéresis de la lesión con bisturí frío, realizando chequeo y control de la hemostasia de forma local mediante compresión principalmente, sin mayores complicaciones de sangrado. Se realizó seguimiento de 3 y 6 meses en donde no se constata recurrencia de la lesión, obteniendo un resultado estético favorable y la satisfacción del paciente.

Se concluye que la presencia del hemangioma lobular capilar en la lengua es una presentación inusual que requiere de un estudio clínico inicial, una detallada historia clínica en donde se consigne el estado sistémico del paciente, índice de higiene oral y factores predisponentes como uso de fármacos como la ciclosporina, los cuales pueden estimular una respuesta exagerada en los tejidos formando un HLC. Lo mencionado anteriormente puede dirigirnos a un diagnóstico presuntivo, el cual confirmaremos siempre con el estudio histopatológico ${ }^{8}$.

\section{Referencias bibliográficas}

1. Jafarzadeh H, Sanatkhani M, \& Mohtasham, N. Oral pyogenic granuloma: a review. J Oral Sci. 2006;48(4):167-75.

2. Boza OYV, Ovares SS. Granuloma piógeno en labio inferior. Reporte de caso y revisión de literatura. Odovtos-Int J Dent Sc. 2016;18(1E):77-84.

3. Díaz LM, Castellanos JL. Lesiones de la mucosa bucal y comportamiento de la enfermedad periodontal en embarazadas. Med. oral patol. oral cir. bucal. 2004;9(5):430-37. 
4. Cordero K, Pardo N, Arellano A. Granuloma piógeno de presentación inusual: Reporte de caso. Odontol Vital. 2016;25:35-42.

5. Parisi E, Glick PH, Glick M. Recurrent intraoral pyogenic granuloma with satellitosis treated with corticosteroids. Oral Dis. 2006;12(1):70-2.

6. Sapp JP, Eversole LR, Wyscoki GP. Patología Oral y Maxilofacial Contemporánea. $1^{\mathrm{a}}$ edición. Madrid (España): Harcourt Brace de Espańa S.A.; 1998. Capítulo 9, Lesiones del tejido conjuntivo; p. 277-318.

7. Ceccotti EL, Sforza RR, Carzoglio JC, Luberti R, Flichman JC. El Diagnóstico en Clínica Estomatológica. 1ª ed. Buenos Aires (Argentina): Editorial Médica Panamericana S.A; 2007. Capítulo 16, Hiperplasias de la mucosa bucal; p. 193-206.

8. Orellana Centeno M, Galvan Torres LJ, Nava Calvillo JF, Orellana Centeno JE. Granuloma piógeno. Presentación de un caso. Odontol Pediátr. 2013;21(2):151-56.

9. Sachdeva SK. Extragingival Pyogenic Granuloma: an Unusual Clinical Presentation. J Dent (Shiraz,Iran). 2015;16(3):282-85.

10. Carrillo-De-Albornoz A, Figuero E, Herrera D, Bascones-Martínez A. Gingival changes during pregnancy: II. Influence of hormonal variations on the subgingival biofilm. J Clin. Periodontol. 2010;37(3):230-40.
11. Yuan K, Wing LYC, Lin MT. Pathogenetic roles of angiogenic factors in pyogenic granulomas in pregnancy are modulated by female sex hormones. J Periodontol. 2002; 73(7):701-8.

12. Reddy NR, Kumar PM, Selvi T, Nalini HE. Management of recurrent post-partum pregnancy tumor with localized chronic periodontitis. Int J Prev Med. 2014;5(5):643-7.

13. Piñas-Caballero L, Pérez-Aguilar M. Granuloma piógeno oral: tratamiento. Cient Dent. 2012;9:33-40.

14. Gadea Rosa C, Cartagena Lay A, La Torre AC. Oral pyogenic granuloma diagnosis and treatment: a series of cases. Rev Odontológica Mex. 2017;21(4):e244-52.

15. Costa FWG, Lima ATT, Cavalcante RB, Pereira KMA. Exuberant pyogenic granuloma in extragingival site. Braz J Otorhinolaryngol. 2012;78(4):134.

16. Parajuli R, Maharjan S. Unusual presentation of oral pyogenic granulomas: a review of two cases. Clin Case Reports. 2018;6(4):690-93.

17. Arunmozhi U, Priya RS, Kadhiresan R, Sujatha G, Shamsudeen-Ss SM. A Large Pregnancy Tumor of Tongue - A Case Report. J Clin Diagn Res. 2016;10(10):10-12.

18. Arunmozhi A, Navabi N, Torabi Parizi M. Unusual Occurrence of Pregnancy Tumor on the Tongue. Iran J Public Health. 2014;43(6):845-46. 